\title{
Coronary heart disease and lipids in women
}

\begin{abstract}
Heart conditions in women are many times disregarded or interpreted as psychological symptoms. In this article we present the data of the high frequency of coronary heart disease as a growing concern in treating women. Coronary ischemic diseases raise a question among epidemiologists regarding menopause being an additional risk factor for ischemic heart disease, since age over 55years in women (age at which women on average have already experienced menopause) and over 45years in men are independent risk factors. It is understood by menopause the deprivation of protective estrogens against atherosclerosis. It is known that atherosclerosis begins in childhood and will usually manifest after 55years in men and 65years in women. Old age is a marker of the amount of established atherosclerotic plaques. The higher the amount of plaques, the higher the risk of ischemic heart disease. Prospective studies with hormone replacement therapy in women without atherosclerosis may clarify the role of estrogen deprivation in atherogenesis.
\end{abstract}

Keywords: women, heart, lipids, hormones, risk factors
Volume I 3 Issue 5 - 2020

\author{
José Mendes Aldrighi,' Anita LR Saldanha,' \\ Ana Paula Pantoja Margeotto,' André Luis \\ Valera Gasparoto, ${ }^{2}$ Tania Leme da Rocha \\ Martinez' \\ 'Department of Nephrology,A Beneficência Portuguesa de São \\ Paulo, Brazil \\ ${ }^{2}$ Department of Intensive Care Unit, Beneficência Portuguesa de \\ São Paulo, Brazil
}

Correspondence: Tania Leme da Rocha Martinez, BP - A Beneficência Portuguesa de São Paulo, Rua Comandante Ismael Guilherme, 358 - Jardim Lusitânia,CEP 0403 I- 120 - São Paulo SP, Brazil,Tel 55 || 98323-9863, Fax 55 || 3842-3789, Email tamar@uol.com.br

Received: September 02, 2020 | Published: October 02, 2020
Abbreviations: AMI, acute myocardial infarction; Apo A-I - Apoprotein A-I; Apo B, apolipoprotein B; CAD, coronary artery disease; CVD, cardiovascular disease; HDL-c, high density lipoprotein cholesterol; VLDL, very low density lipoprotein

\section{Mini review}

In women, $46 \%$ of deaths result from cardiovascular disease (CVD) and $50 \%$ of them refer to coronary heart disease.

Women, more than men, have angina pectomy as the initial manifestation of the disease ( $65 \%$ vs. $35 \%$, respectively). Men, on the other hand, have acute myocardial infarction (AMI) as an initial manifestation in a higher proportion than in women $(29 \%$ vs. $43 \%$, respectively). The varied initial manifestations of ischemic diseases result in different diagnostic approaches; thus, as women report milder symptoms, despite a more severe disease, they are less submitted to diagnostic procedures, even having a positive stress test and, when they are, they are already in a more advanced state of the disease. ${ }^{1-11}$

Once coronary heart disease is diagnosed in women, the rate of fatal cases exceeds that of men. Although most recurrent AMI occurs in men at a rate of $80 \%$ (perhaps because women are more likely to die after their first episode), the rate of reinfarction in women is also high. During the first 3 to 4 years after AMI, approximately $20 \%$ of women will have a repeat of the episode.

The fact that women in middle age or older women are at higher risk for CVD is particularly true after the onset of menopause. Observations made by several authors ${ }^{1}$ indicate that, in addition to age, there would be an additional risk factor that would increase the chance of CVD. Thus, it was speculated that this factor would be estrogenic deficiency that begins gradually a few years before the onset of menopause. Women with early menopause have increased risk for CVD similar to those of postmenopausal, and those in postmenopausal periods undergoing hormone replacement therapy show a decrease in risk for CVD when compared to the untreated group.

\section{Risk factors}

In the Framingham longitudinal study, ${ }^{1}$ 2,873 women were followed for a period of 30years, and of these, 574 developed a clinical picture of coronary artery disease (CAD). Stable angina was present in 315 women, AMI in 195, unstable angina in 51, and sudden death in 73 . The incidence of CAD was directly related to the presence of several risk factors, some of which were: dyslipidemia, glucose intolerance, hyperuresia, hyperfibrinogenemia and obesity. In the multivariate analysis for any total cholesterol level, the total cholesterol ratio/HDL-c (High Density Lipoprotein Cholesterol) identified the population at greatest risk. When this ratio is greater than 5 , the risk of CAD is approximately three times greater than a desirable ratio of 3.5 .

Other data from the Framingham study showed that total serum cholesterol is a primary risk factor also in people over 65years of age; for every $1 \%$ increase in total cholesterol, coronary heart disease increases by $2 \%$ to $3 \%$. LDL-c (Low Density Lipoprotein Cholesterol), as a predictor of coronary heart disease, is no better index than total cholesterol; however, the total cholesterol/HDL-c ratio (Risk Index I) predicts better than HDL-c or only total cholesterol in both sexes.

The Lipid Research Clinics Study ${ }^{1}$ followed 1,405 women over 50 and under 69 for an average period of 14years. This study found a strong association between HDL-c levels $<50 \mathrm{mg} / \mathrm{dL}$ and CAD mortality. Triglyceride levels greater than $200 \mathrm{mg} / \mathrm{dL}$ correlate with increased mortality from CAD, especially if associated with low HDL-c levels. It was also observed that in HDL-c women $<$ than $50 \mathrm{mg} / \mathrm{dL}$ and triglycerides $>$ that $200 \mathrm{mg} / \mathrm{dL}$, the mortality rate due to CAD was 8 times higher than the rate of women who had low HDL-c levels and normal triglyceride levels. On the other hand, patients with elevated HDL-c levels with triglycerides above normal had three times more coronary events than those with normal triglycerides. The analysis of total cholesterol showed that, for any level of total cholesterol, mortality was higher in patients with low HDL-c, and that regardless of HDL-c, an increase in the rate of events was evidenced only when the total cholesterol values were higher than $240 \mathrm{mg} / \mathrm{dL}$. 
The Donolo-Tel Aviv Study ${ }^{1}$ followed 2,800 men and women for a 20-year period and demonstrated an increase in CAD mortality of 2 to 5 times in the group of women, who had serum HDL-c levels that corresponded to less than $23 \%$ of total cholesterol levels.

From the studies presented, we can observe that HDL-c is one of the factors of great importance in the risk of developing CAD in women. Even in those cases where serum LDL-c or total cholesterol is above normal values, high HDL-c levels can contribute significantly to minimizing risk. In the 1993 American Consensus, ${ }^{1}$ this antiatherogenic fraction is identified as a negative risk factor when its levels are higher than $60 \mathrm{mg} / \mathrm{dL}$. From the total sum of risk factors should be subtracted 1 and, from the result, the treatment schedule is established. ${ }^{12-14}$

Total serum cholesterol levels, higher than $240 \mathrm{mg} / \mathrm{dL}$, behave as an independent risk factor in several studies. ${ }^{1}$ In women, triglycerides seem to participate as an independent risk factor for the development of CAD in cases of association with low HDL-c.

The identification of risk factors, highlighted below, allows systematic treatment of the at-risk population (hyperlipidemia, diabetes mellitus, and hypertension) and the primary prevention of that, with changes in lifestyle (eating habits, smoking and alcohol).

\section{Main cardiovascular risk factors}

a. Age - Men $\geq 45$ years- Women $\geq 55$ years or early menopause without hormone replacement

b. Family history of premature coronary disease (defined myocardial infarction or sudden death before 55years of age in father or other men, first-degree relatives, or before 65years of age in mother or other women first-degree relatives)

c. Smoking

d. Systemic arterial hypertension

e. High LDL-cholesterol

f. HDL-cholesterol $<35 \mathrm{mg} / \mathrm{dL}$

g. Diabetes mellitus

h. Negative Risk Factor

i. HDL-cholesterol $\geq 60 \mathrm{mg} / \mathrm{dL}$

j. Special situations: Lipid changes during the menstrual cycle

It is to be assumed that during the menstrual cycle, when there are major hormonal changes in the woman, there is some change in the lipid profile. It has been demonstrated that in the estrogen phase or first period of the cycle, there is a progressive fall in LDL-c, which continues until the end of this phase, returning to baseline values in the period in which progesterone is most produced. The same author showed no alteration in HDL-c or triglycerides. ${ }^{1}$

Woods et al. ${ }^{1}$ conducted a similar study and found a $3 \%$ increase in triglycerides during the estrogenic phase of the cycle, showing no alterations of HDL-c or LDL-c. ${ }^{1}$

The reason why there was no drop in HDL-c levels in the estrogen phase was not clarified; it is possible that the time required for this hormone to determine some change in this lipoprotein is a little longer, such as when it occurs in postmenopausal, in which, with hormone replacement, the fall is expected around 4-6months. Lipid changes in pregnancy. The plasma concentration of all lipoproteins increases substantially during pregnancy. We owe this to the increase in estrogen and progesterone, which, in this period, reach respectively 16 and 7 times the normal values, in the term period.

Triglycerides increase by two to three times the normal values, reaching the peak in the term phase with a progressive return to baseline values, which may take until the end of the first month of puerperium. The behavior of total cholesterol levels is similar to that of triglycerides, increasing progressively, reaching two to five times the normal values in the term phase, with a slightly slower decrease than triglyceride levels. Its normalization may take up to 6weeks after delivery. As for lipoprotein fractions, they also present qualitative alterations; HDL-c and LDL-c have increased amount of triglycerides.

HDL-c has a slightly different behavior from total cholesterol and triglycerides, as it presents a progressive increase reaching the maximum in the $24^{\text {th }}$ week, with an increase of $50 \%$ in values when compared with non-pregnancy period, and then presents a drop being $15 \%$ above normal values (non-pregnancy period) until the term period.

LDL-c levels increase in synchronism with those of total cholesterol, but show a more delayed decrease, and the fall may occur after the eighth week of puerperium. The factor responsible for these lipoprotein changes is the hormone estrogen. What would explain the drop in HDL-c after the $24^{\text {th }}$ week would be the increase in plasma insulin concentration, which represents an increase in insulin resistance. It is concluded, therefore, that HDL-c levels are more related to estrogen level in the first phase of pregnancy and more related to insulin in the second phase. It is recommended that the dosage of a lipid profile be postponed at least 4 to 6 months after pregnancy, especially in those women without previous alterations. ${ }^{2}$

\section{Lipid changes with oral contraceptive use}

Several studies have demonstrated the adverse effects of oral contraceptive use on serum lipid levels. ${ }^{2}$ It is also known that the interference in lipid profile depends a lot on the amount, type and dose of the different hormone presentations, which exist in the composition of contraceptives. Currently, there is a consensus that lipid changes induced by contraceptives are not alone responsible for the increased risk of a cardiovascular event, but rather association with changes in coagulation parameters, blood pressure and the presence of other risk factors.

Of the studies conducted regarding the alteration of the lipid profile secondary to the use of contraceptives, the most considered are those whose follow-up was longer, with different compositions of contraception, and with a greater number of patients.

The Framingham Study ${ }^{3}$ followed a total of 1,930 women of any age group. Of these, 992 were premenopausal, and 57 were on contraceptive use. Evaluating the lipid profile showed for any hormonal dosage an increase in serum levels of total cholesterol and triglycerides. It demonstrated an increase of around 10\% in HDL-c levels in women with various forms of contraceptive, finding this concordant with some and discordant from other studies. ${ }^{3}$ When evaluating lipid alterations in relation to hormonal composition, this study demonstrated that the changes are related to the amount of estrogen and progestagenos. Women using contraceptives with higher estrogen had lower serum levels of LDL-c and apo B (Apolipoprotein 
B), and in those where progestagenos dosage was higher, lower levels of HDL and apo A-I (Apoprotein A-I) were found. However, analyzing the total cholesterol/HDL-c ratio, it was verified, for any hormonal dosage, that there was no change in this relationship that could be classified as atherogenic, and this relationship was always between 3 and 4 .

Comparative study of 190 women, ${ }^{3}$ divided into two groups: women using contraceptives, and women who had used contraceptives in the three years prior to the study and who were not in current use, demonstrated that women belonging to the first group had a significant increase in triglyceride levels $(95 \times 73 \mathrm{mg} / \mathrm{dL})$ and a trend of significance with total cholesterol ratio in these two groups (198 x $189 \mathrm{mg} / \mathrm{dL})$, the same as with serum HDL levels $(47$ x $50 \mathrm{mg} / \mathrm{dL})$.

Meade et al. $^{3}$ for 12 months, accompanied women using various forms of contraceptives. Several compositions were associated, but with the same dose of estradiol $(0.05 \mathrm{mg})$, with an increase of 10 to $15 \%$ in LDL-c levels, and when this estradiol dosage was associated with levonogestrel at a dose of $0.25 \mathrm{mg}$, a drop in serum HDL-c levels of $13 \%$. When they used the association with etinodiol acetate at a dose of $1 \mathrm{mg}$, there was a $1 \%$ decrease in HDL-c levels; already using the combination with noretindrone at a dose of $1.0 \mathrm{mg}$, there was an increase in serum HDL-c levels of 3\%. The norgestrel, because it is the most androgenic of the progestagenos and because it does not undergo the phenomenon of the first passage, exerts a strong effect on the uptake and lipoprotein metabolism of the liver, causing the greatest alterations. ${ }^{3}$

It has been demonstrated, both experimentally and clinically, that the use of contraceptives interferes in a deleterious way in coagulation. There was a positive association between administered dose of estrogen with increased serum levels of factor VII-C and fibrinogen, and these, in turn, have been associated with a higher incidence of ischemic heart disease. On the other hand, contraceptive use with high plasmin levels, which activates plasminogen, was associated with an effect contrary to the procoagulant effect. This could be an adaptive change to the procoagulant changes described at the beginning.

Analyzing these studies, we can state that: the changes in lipid profile induced by estrogens and progestagens are quite different. The effects depend on the type, dose and composition of the different preparations. The use of oral contraceptives is related to lipoprotein changes by direct effect on the liver. The development of new formulations, in which progestagen doses are less androgenic and do not affect the lipid profile negatively, makes contraception very safe.

It is recommended that, before starting contraception, the woman be submitted to a lipid profile analysis. We should be aware of HDL-c levels below $35 \mathrm{mg} / \mathrm{dL}$, and also with high triglyceride levels, especially in those where there is an association with other risk factors such as smoking, family history, and hypertension. Therefore, a dosage is recommended before starting contraception, and, after its onset, a semiannual dosage in the first year, and from the second year of use, annual dosages. ${ }^{15}$

\section{Lipid changes in postmenopausal}

Plasma lipids and lipoproteins are important risk factors for the development of atherosclerotic CVD in menopausal women. Several situations that occur in the postmenopausal period can determine changes in lipid profile, such as obesity, sedentary lifestyle and hormonal changes. In postmenopausal periods, there are increases in cholesterol, triglycerides and LDL-c levels, and HDL-c levels remain relatively constant. The fraction of HDL-c that causes the strongest changes during the postmenopausal period is the $\mathrm{HDL}_{2}$ fraction and it is admitted that it is the most important in the prevention of atherosclerotic disease.

Treatment with estrogens is accepted as having fundamental and important action on lipoprotein metabolism, promoting favorable changes in lipid profile. Several studies ${ }^{3}$ have demonstrated the beneficial influence of hormone replacement therapy in postmenopausal on plasma lipid concentration. The results of these studies $^{3}$ reflect a large number of variations that depend on the population studied hormonal formulation, treatment regimens and duration of use. Estrogen treatment promotes favorable changes in lipid profile, including increased levels of HDL-c (and its $\mathrm{HDL}_{2}$ and $\mathrm{HDL}_{3}$ fractions) and decreased LDL-c levels.

Intrahepatic mechanisms of estrogenic action, which explain these changes, include:

1. Increase in catabolism and clearance of LDL-c, by increasing the number of receptors in hepatocytes and the production of VLDL (Very Low Density Lipoprotein) of long chain, which are metabolized directly from plasma;

2. the decrease in liver receptors for HDL-c and, therefore, reduction of their clearance, the significant reduction in lipase activity that contribute to further decrease HDL-c clearance and increased apoproteins A-I and A-II that induce an increase in HDL-c production;

3. inhibition of the production of regulatory oxysteroids;

4. Inhibition of cholesterol catabolism to bile acids, leading to increased liver exposure to free cholesterol. Therefore, bile secretion of cholesterol is increased and the total effect is the reduction of cholesterol accumulation in peripheral tissues and its increase in bile fluid;

5. increased clearance of chylomicrons. It should be emphasized that the alterations of the lipid profile are dose-dependent and the estrogenic effects on lipids and lipoproteins are greater when they are used orally.

And what are the effects of progestogens on lipid metabolism? Initially, it is important to consider that postmenopausal women treated with estrogen replacement are usually supplemented with cyclic or continuous progestogens, in order to block the estrogenic effect on the endometrium. Since progesterone and other progestogens are antagonists of estrogenic action, their use could reduce the beneficial effects of estrogen on the cardiovascular system. Thus, in relation to lipoprotein levels, natural progestogens do not seem to cause significant changes. On the other hand, synthetic progestogens, especially those with androgenic activity, can exert significant metabolic effects, such as increased LDL-c levels and decreased HDL-c levels, particularly its $\mathrm{HDL}_{2}$ fraction.

Derived C-21 progestogens, such as medroxyprogesterone acetate, represent the best choice because they have less impact on lipid profile than 19-nor-testosterone derivatives, which have significant adverse effects on plasma lipids, even neutralizing the beneficial effects of estrogens. The mechanisms by which progestogens can alter the lipid profile are not yet well known, but are known to be related to increased hepatic lipase. It should be considered that the effects of progestogens on lipid profile are dose-dependent, that is, the higher the dose, the greater the impact on lipoproteins; in relation to the metabolic effects 
of the addition of progestogens to Hormone Replacement Therapy are dependent on the concomitant dose of estrogens.

\section{Hormone replacement therapy}

This subject is considered one of the most important to address the field of dyslipidemia therapy in women. Mainly because it is a theme that characterizes the approach of postmenopausal women with higher risk. In addition to being current in the treatment of women, the proven beneficial effects consolidate its value.

Studies have demonstrated ${ }^{3}$ the undesirable changes that occur in the lipid profile of postmenopausal women. On the other hand, it was too demonstrated that hormone replacement for this group of women would act positively in this profile, which would be translated by lower risk of developing CVD. Hormone replacement positively alters lipid profile, leading to a decrease in total cholesterol and LDL-c levels, and increased HDL-c. The major problem of longterm hormone replacement concerns an increase in the incidence of endometrial hyperplasia when it is performed alone with estrogen. Hormone replacement therapy with estrogens showed benefits in reducing $(50 \%)$ coronary events and death by CAD in patients who were users. Recent study ${ }^{3}$ in which 875 women were randomized into five treatment groups (I - placebo, II - CEE conjugated equine estrogen- $0.625 \mathrm{mg} /$ day/continuous, III - CEE $0.625 \mathrm{mg} /$ day/ continuous associated with medroxyprogesterone acetate MPA $10 \mathrm{mg}$ day $/ 12$ days, IV - CEE- $0.625 \mathrm{mg} /$ day/continuous associated with continuous MPA $2.5 \mathrm{mg}, \mathrm{V}$ - CEE $0.625 \mathrm{mg} /$ day/continuous associated with micronized progesterone $200 \mathrm{mg}$ /day for 12 days of the cycle) and monitored for threeyears, with the main objective of, at the end of this period, study whether the interference of hormone replacement on four risk factors: total cholesterol, serum fibrinogen, insulinemia and blood pressure, demonstrated that when the woman is hysterectomized, the best form of replacement is with estrogen alone (CEE-0 increase by 10 to $15 \%$ in HDL-c levels. In non-hysterectomized patients, the best regimen would be an association of estrogen with a progestagene, whether micronized progesterone $200 \mathrm{mg}$ /day during 12 days of the cycle, or medroxyprogesterone acetate $10 \mathrm{mg}$ day during the first 10days of the cycle; this association aims to reduce the risk of the installation of uterine cancer. It is recommended, therefore, for these patients in hormone replacement therapy, a close follow-up on the part of the gynecologist, with the objective of controlling and preventing changes of this type.

\section{Conclusion}

Hormone replacement in the PEPI study was shown to be effective in reducing total cholesterol $(20 \%)$, LDL-c $(20 \%)$, promoting an increase in HDL-c (10\%), not altering insulinemia or fasting glycemia, and also did not lead to changes in blood pressure levels or caused weight gain. Postmenopausal patients with serum lipid changes, other risk factors for Cad, risk for developing osteoporosis or patients with coronary arteriopathy should be the major beneficiaries of hormone replacement, both prophylactic and therapeutic.

Randomized studies are underway with the aim of demonstrating that hormone replacement in postmenopausal women will be able to reduce $\mathrm{CAD}$ death rates. ${ }^{16}$

\section{Conflicts of interest}

No conflict of interest.

\section{Funding}

None.

\section{References}

1. Barros MAV, Martinez TLR, Cendoroglo MS. Metabolic and therapeutic aspects of dyslipidemias associated with cardiovascular disease. In: Lima GR, Baracat EC, editors. Endocrine gynecology. Atheneu. 2nd edn. Rio de Janeiro: Atheneu; 1995. p. 282-291.

2. Avila WS, Alexandre ERG, Castro ML, et al. Brazilian Cardiology Society Statement for Management of Pregnancy and Family Planning in Women with Heart Disease - 2020. Arq Bras Cardiol. 2020;114(5):849-942.

3. Aldrighi JM, Martinez TLR, Silva Filho CP. Cardiovascular disease and hormone replacement therapy. Arq Bras Cardiol. 1995;65(3):249-254.

4. Kannel WB, Vokonas PS. Demographics of the prevalence, incidence, and management of coronary heart disease in the elderly and in women. Ann Epidemiol. 1992;2(1-2):5-14.

5. Solimene MC. Coronary heart disease in women: a challenge for the $21 \mathrm{st}$ century. Clinics (Sao Paulo). 2010;65(1):99-106.

6. Ashley KE, Geraci SA. Ischemic heart disease in women. South Med J. 2013;106(7):427-433.

7. Biddle C, Fallavollita JA, Homish GG, et al. Gender differences in symptom misattribution for coronary heart disease symptoms and intentions to seek health care. Women Health. 2020;60(4):367-381.

8. Bulliyya G. Risk of coronary heart disease in women after menopause. $J$ Indian Med Assoc. 2001;99(9):478-480.

9. Galick A, D’Arrigo-Patrick E, Knudson-Martin C. Can anyone hear me? Does anyone see me? A qualitative meta-analysis of women's experiences of heart disease. Qual Health Res. 2015;25(8):1123-1138.

10. Mozaffarian D, Benjamin EJ, Go AS, et al. Heart disease and stroke statistics - 2015 update: a report from the American Heart Association. Circulation. 2015;131(4):e29-322.

11. Bucholz EM, Butala NM, Rathore SS, et al. Sex differences in long-term mortality after myocardial infarction: a systematic review. Circulation. 2014;130(9):757-767.

12. Wilmot KA, O'Flaherty M, Capewell S, et al. Coronary heart disease mortality declines in the United States from 1979 through 2011: evidence for stagnation in young adults, especially women. Circulation. 2015;132(11):997-1002.

13. Mansur AP, Gomes EPSG, Avakian SD, et al. Clustering of traditional risk factors and precocity of coronary disease in women. Int $J$ Cardiol. 81(2-3):205-209.

14. Aldrighi JM. Oral hormonal contraception and thromboembolism? Rev Assoc Med Bras. 2003;49(2):120.

15. Saltiki K, Alevizaki M. Coronary heart disease in postmenopausal women; the role of endogenous estrogens and their receptors. Hormones (Athens). 2007;6(1):9-24.

16. Villa P, Amar ID, Shachor M, et al. Cardiovascular risk/benefit profile of MHT. Medicina (Kaunas). 2019;55(9):571.

\section{Acknowledgments}

None. 